\title{
Remote Assessment of Disease and Relapse in Major Depressive Disorder (RADAR-MDD): Recruitment, retention, and data availability in a longitudinal remote measurement study.
}

Faith Matcham ( $\nabla$ faith.matcham@kcl.ac.uk)

Institute of Psychiatry, Psychology and Neuroscience, King's College London

Daniel Leightley

Institute of Psychiatry, Psychology and Neuroscience, King's College London

Sara Siddi

Parc Sanitari Sant Joan de Déu, Fundació Sant Joan de Déu, CIBERSAM, Universitat de Barcelona

Femke Lamers

Department of Psychiatry and Amsterdam Public Health Research Institute, Amsterdam UMC, Vrije Universiteit

\section{Katie M White}

Institute of Psychiatry, Psychology and Neuroscience, King's College London

\section{Peter Annas}

H. Lundbeck A/S

\section{Giovanni de Girolamo}

IRCCS Instituto Centro San Giovanni di Dio Fatebenefratelli

\section{Sonia Difrancesco}

Department of Psychiatry and Amsterdam Public Health Research Institute, Amsterdam UMC, Vrije Universiteit

\section{Josep Maria Haro}

Parc Sanitari Sant Joan de Déu, Fundació Sant Joan de Déu, CIBERSAM, Universitat de Barcelona Melany Horsfall

Department of Psychiatry and Amsterdam Public Health Research Institute, Amsterdam UMC, Vrije Universiteit

\section{Alina Ivan}

Institute of Psychiatry, Psychology and Neuroscience, King's College London

\section{Lavelle Grace}

Institute of Psychiatry, Psychology and Neuroscience, King's College London

\section{Qingqin Li}

Janssen Research and Development, LLC

\section{Federica Lombardini}


Parc Sanitari Sant Joan de Déu, Fundació Sant Joan de Déu, CIBERSAM, Universitat de Barcelona

\section{David C Mohr}

Center for Behavioral Intervention Technologies, Department of Preventative Medicine, Northwestern University

\section{Vaibhav A Narayan}

Janssen Research and Development, LLC

\section{Carolin Oetzmann}

Institute of Psychiatry, Psychology and Neuroscience, King's College London

\section{Brenda BWJH Penninx}

Department of Psychiatry and Amsterdam Public Health Research Institute, Amsterdam UMC, Vrije Universiteit

\section{Stuart Bruce}

RADAR-CNS Patient Advisory Board, King's College London

\section{Nica Raluca}

RADAR-CNS Patient Advisory Board, King's College London

\section{Sara K Simblett}

Institute of Psychiatry, Psychology and Neuroscience, King's College London

\section{Til Wykes}

Institute of Psychiatry, Psychology and Neuroscience, King's College London

\section{Jens C Brasen}

H. Lundbeck A/S

\section{Inez Myin-Germeys}

Department for Neurosciences, Center for Contextual Psychiatry

\section{Aki Rintala}

Faculty of Social and Health Care, LAB University of Applied Sciences

\section{Pauline Conde}

Institute of Psychiatry, Psychology and Neuroscience, King's College London

\section{Richard JB Dobson}

Institute of Psychiatry, Psychology and Neuroscience, King's College London

\section{Amos A Folarin}

Institute of Psychiatry, Psychology and Neuroscience, King's College London

\section{Callum Stewart}

Institute of Psychiatry, Psychology and Neuroscience, King's College London

\section{Yatharth Ranjan}

Institute of Psychiatry, Psychology and Neuroscience, King's College London

\section{Zulqarnain Rashid}

Institute of Psychiatry, Psychology and Neuroscience, King's College London

\section{Nick Cummins}

University of Augsburg 
Nikolay V Manyakov

Janssen Pharmaceutica NV

Srinivasan Vairavan

Janssen Research and Development, LLC

Matthew Hotopf

Institute of Psychiatry, Psychology and Neuroscience, King's College London

\section{Research Article}

Keywords: Major Depressive Disorder, Remote Measurement Technologies, longitudinal, multicentre, cohort study

Posted Date: June 14th, 2021

DOl: https://doi.org/10.21203/rs.3.rs-612374/v1

License: (c) (i) This work is licensed under a Creative Commons Attribution 4.0 International License.

Read Full License

Version of Record: A version of this preprint was published at BMC Psychiatry on February 21st, 2022.

See the published version at https://doi.org/10.1186/s12888-022-03753-1. 


\section{Abstract \\ Background}

Major Depressive Disorder (MDD) is prevalent, often chronic, and requires ongoing monitoring of symptoms to track response to treatment and identify early indicators of relapse. Remote Measurement Technologies (RMT) provide an exciting opportunity to transform the measurement and management of $M D D$, via data collected from inbuilt smartphone sensors and wearable devices alongside app-based questionnaires and tasks. Our aim is to describe the amount of data collected during a multimodal longitudinal RMT study, in an MDD population.

\section{Methods}

The Remote Assessment of Disease and Relapse - Central Nervous System (RADAR-CNS) program explores the potential to use RMT across a range of central nervous system disorders. Remote Assessment of Disease and Relapse - Major Depressive Disorder (RADAR-MDD) is a multi-centre, prospective observational cohort study conducted as part of the RADAR-CNS program. People with a history of MDD were provided with a wrist-worn wearable, and several apps designed to: a) collect data from smartphone sensors; and b) deliver questionnaires, speech tasks and cognitive assessments. Participants were followed-up for a maximum of 2 years.

\section{Results}

A total of 623 individuals with a history of MDD were enrolled in the study. We report $80 \%$ completion rates for primary outcome assessments across all follow-up timepoints. $79.8 \%$ of people participated for the maximum amount of time available and $20.2 \%$ withdrew prematurely. Data availability across all RMT data types varied depending on the source of data and the participant-burden for each data type. We found no evidence of an association between the severity of depression symptoms at baseline and the availability of data. In total, 110 participants had $>50 \%$ data available across all data types, and thus able to contribute to multiparametric analyses.

\section{Conclusions}

RADAR-MDD is the largest multimodal RMT study in the field of mental health. Here, we have shown that collecting RMT data from a clinical population is feasible. We found comparable levels of data availability in active and passive forms of data collection, demonstrating that both are feasible in this patient group. Our next steps are to illustrate the predictive value of these data, which will be the focus of our future data analysis aims. 


\section{Background}

Depressive disorders contribute to $14.3 \%$ of all-age years lived with disability (YLD), making it the third leading cause of YLD globally (1). Major depressive disorder (MDD) is a severe form of depression characterised by prolonged periods of low mood and anhedonia combined with a range of other symptoms including changes in sleep quality, appetite, cognitive function, energy levels, activity, feelings of guilt or worthlessness and thoughts of death (2). MDD is associated with a wide range of negative outcomes including: loss of occupational function (3); reduced quality-of-life (4); and premature mortality (5). Whilst some may experience a single lifetime episode of MDD, it is becoming more widely recognised as a chronic condition, characterised by periods of relapse and recovery $(6,7)$. The management of chronic illnesses requires ongoing monitoring of symptoms to track response to treatment and identify early indicators of relapse. Conventionally, this monitoring is dependent on selfreported questionnaires or clinical interviews, which are reliant on individuals' subjective recollection of symptoms. This is subject to recall bias and loses context reactivity and symptom variability (8). That is, how symptoms change in relation to situational factors (context reactivity) and how symptoms may fluctuate on a daily, or even hourly basis (symptom variability).

The use and ownership of smartphones and wearable technology has increased exponentially in the last decade; new technological advances facilitate the collection of digital biomarkers using unobtrusive, inbuilt sensors requiring minimal input from users $(9,10)$. Many features of MDD, including symptoms, risk factors for, and consequences of, depression are amenable to assessment via remote measurement technologies (RMT): for example, heart rate from photoplethysmography (PPG) sensors and activity from accelerometery sensors in wrist-worn wearable devices can give information indicative of sleep patterns and physical activity levels. Data such as Global Positioning System (GPS), Bluetooth, gyroscope, phone screen interactions, ambient noise and light levels have also been used to unobtrusively collect information from smartphones relating to sociability, movement and activity associated with low mood (11). In contrast to this passive RMT (pRMT) form of data collection, requiring little or no input from the user, active RMT (aRMT), requires action from the user, such as completing short questionnaires or tasks in response to a notification. Combining data collected passively via inbuilt smartphone sensors, with smartphone apps to deliver questionnaires, cognitive tasks, assessments of speech or Experience Sampling Method (ESM) we can gain valuable insight into an individual's daily life (12).

There is a growing body of research studies using these technologies to measure symptoms and predict outcomes (11), or to deliver interventions in MDD (13). However, the real-world implementation of digital health applications into clinical care has been thwarted by low uptake, adherence and retention; despite the availability of over 300,000 health apps and more than $\$ 9$ billion in investment (14). Reports from industry suggest that in $2019,25 \%$ of health apps are opened only once, and only $32 \%$ of users use a health app more than 10 times (15).

Initial uptake and continued health app use in people with depression may be even lower, with systematic review evidence suggesting that long-term ( $\geq 6$ weeks) use may be as low as $0.5 \%$ (16). Digital exclusion 
is prevalent in people with mental disorders: reduced access to technology; unpredictable personal circumstances and symptoms of the mental disorder itself which may affect cognitive functions and confidence have all been associated with a lack of engagement with digital technologies (17). Beyond initial uptake, longer-term use of health apps in people with depression is affected by a complex combination of health-, user- and technology-related barriers (18). There is, however, a widely acknowledged ambiguity in the reporting of engagement statistics and a huge amount of variation in the methods used to assess uptake and engagement in digital technology research $(19,20)$.

The Remote Assessment of Disease and Relapse - Central Nervous System (RADAR-CNS; (21) program is an international consortium exploring the potential for RMT to transform the measurement and management of MDD, epilepsy and multiple sclerosis. Remote Assessment of Disease and Relapse in Major Depressive Disorder (RADAR-MDD; (22)) is a longitudinal cohort study being conducted as part of the RADAR-CNS program, examining the utility of multi-parametric RMT to measure changes in symptoms and predict relapse in people with MDD. The study has been designed with patient involvement from the outset (including systematic reviews (23), focus groups (18) and a creating a dedicated Patient Advisory Board) with the aim of developing a protocol which meets the needs of the target population. In addition to its primary purpose of identifying RMT predictors of depression outcomes, RADAR-MDD offers a unique opportunity to explore the recruitment of people with MDD into a complex digital technology study, and describe the long-term retention rates and adherence to a protocol which includes passive data collection via smartphone and wearable sensors, app-based questionnaires, ESM and traditional web-based outcome assessments (22).

Throughout this paper, we have used the term data "availability" instead of "completeness" as we describe all data provided throughout the study, regardless of quality or completeness. Data labelled as "available" in this paper may include i) complete, valid data which are usable for analysis; ii) partial data which are incomplete but potentially usable; and iii) data which have been corrupted or are invalid for any reason. We believe it is essential to include partial or incomplete data as part of this paper, as they are indicative not only of participant characteristics and study burden, but also of the underlying technical infrastructure. It is also essential to note that whilst some participants were withdrawn from the study as "lost to follow-up" for not completing primary outcome assessments, we decided to not withdraw participants for not providing data via the smartphone apps or wearable devices. This concession gives greater insight into how data availability may fluctuate with changes in depressive state, and a truer representation of the feasibility of implementing RMT protocols in people with MDD.

The aims of this paper are to: 1) summarise study recruitment, retention, and completion rates of primary and secondary participant-reported outcomes throughout the course of follow-up; 2 ) describe the sociodemographic and clinical characteristics of the cohort for the RADAR-MDD study; and 3) describe the availability of data throughout a multi-parametric RMT study protocol including active and passive assessments of symptoms, behaviour and cognitive function.

\section{Methods}




\section{Study Design}

The full protocol for RADAR-MDD has been reported elsewhere (22). In short, RADAR-MDD is a multicentre, prospective observational cohort study. The study aimed to examine whether data collected via multiparametric RMT can be used to reliably track illness course and predict relapse in MDD. The study sought to recruit 600 individuals with a recent history of MDD and follow them up for a maximum of 24 months. The study involved no randomisation, intervention, or allocation of people into groups. The study has three recruitment sites: King's College London (KCL; UK); Amsterdam University Medical Centre, location VUmc (Amsterdam, The Netherlands); and Centro de Investigación Biomédica en Red (CIBER; Barcelona, Spain).

\section{Study Population}

To be eligible for participation in RADAR-MDD, individuals must: 1) have met DSM-5 diagnostic criteria for non-psychotic MDD within the past 2 years; 2) have recurrent MDD (having had a lifetime history of at least 2 episodes); 3) be able and willing to complete self-reported assessments via smartphone; 4) be able to give informed consent; 5) be fluent in English, Dutch, Spanish or Catalan; 5) have an existing Android smartphone, or willingness to swap to Android as their only phone; 6) be aged 18 or over.

Exclusion criteria were: 1) having a lifetime history of bipolar disorder, schizophrenia, MDD with psychotic features, or schizoaffective disorder; 2) dementia; 3) history of moderate or severe drug or alcohol use in the 6 months prior to enrolment; 4) a major medical diagnosis which might impact an individual's ability to participate in normal daily activities for more than two weeks; 5) pregnancy (although once enrolled, becoming pregnant did not result in withdrawal).

Eligible participants were identified via several recruitment channels, including through existing research cohorts who have consented to be contacted for future research opportunities, through primary and secondary mental health services, or through advertisements for the study placed on mental health charity websites, circulars or Twitter notices(22). Participants in Amsterdam were partially recruited through Hersenonderzoek.nl (https://hersenonderzoek.nl/). All participants provided written consent and provided detailed baseline assessments including sociodemographic, social environment, medical history, medical comorbidities and technology use questionnaires, alongside depression history measured via the Lifetime Depression Assessment - Self-Report (LIDAS; (24)).

\section{Data Collection}

\section{Remote Data Collection}

Data collection started in London (UK) in November 2017 in a pilot phase of app development, with additional assessments being added to the protocol throughout the first 18 months of the study period to 
allow small-scale functionality testing and quality control before international large-scale data collection commenced. Data collection started in Barcelona and Amsterdam in September 2018 and February 2019, respectively. A detailed description of the recruitment procedures across sites and schedule of observations has previously been published (22). The data collected used RADAR-base, an open source platform designed to leverage data from wearables and mobile technologies (25). RADAR-base provides both passive and active data collection via two apps - the RADAR active and passive monitoring apps.

\section{Passive RMT}

The passive RMT (pRMT) app unobtrusively collected information about phone usage throughout participation, requiring no input from the participant. It collected data on ambient noise, ambient light, location, app usage, Bluetooth connectivity, phone usage, and battery life. Whilst some measurements were added later, some data sources were also removed from the protocol throughout follow-up (summarised in additional file 1), due to either technical changes or feasibility. For example, the original protocol aimed to collect data about SMS and call logs, however changes to Google's Play Store permissions prevented access to this data as of January 2019. Data pertaining to SMS and call logs have not been reported in the current paper due to data collection from this sensor ceasing whilst one site had only recruited 30 individuals and another site had not started recruitment at all.

Participants were additionally required to wear a Fitbit Charge 2/3 device for the duration of participation, providing information about individuals' sleep and physical activity levels. Participants could keep the Fitbit at the end of the time in the study.

\section{Active RMT}

The RADAR-base active RMT (aRMT) app administered validated measurements of depression and selfesteem every 2-weeks via the 8-item Patient Health Questionnaire (PHQ8; (26)) and Rosenberg SelfEsteem Scale (RSES; (27)). Items on the PHQ8 can be totalled and used as a continuous score with higher scores indicating increased depression severity, and scores totalling $\geq 10$ indicating those with significant symptoms (26). The RSES requires reversing of 5 of the 10 items, which then can be totalled to create a total score with higher scores representing increased self-esteem (27).

The aRMT app also delivered a speech task every 2-weeks, requesting participants to record a predetermined text from the "North Wind and the Sun", which is phonetically balanced across all three languages (28) and answer a question relating to plans for the upcoming week. Finally, the aRMT app included an ESM protocol (22), requiring participants to complete brief questions relating to mood, stress, sociability, activity and sleep, multiple times per day for 6 days at scheduled times throughout the course of follow-up.

\section{Cognitive Function}

Cognitive function was measured every 6-weeks via an additional THINC-it app $\circledast$, which was integrated into the RADAR-base platform. The app has been validated to identify cognitive dysfunction within the 
context of depressive disorder (29). The app contains the 5-item Perceived Deficits Questionnaire (PDQ5; (30)), alongside computerised versions of the Choice Reaction Time Identification Task ("Code Breaker"), One-Back Test ("Spotter"), Digit Symbol Substitution Test ("Symbol Check") and Trail Making Test-Part B ("Trails") tasks to assess processing speed, working memory, concentration and attention (29).

\section{Primary and Secondary Outcome Assessments}

All primary and secondary outcome measurements were collected via automatic surveys sent every 3 months via the Research Electronic Data Capture (REDCap) software (31). A full description of the outcome assessment schedule is provided in our published protocol paper (22).

\section{Depression}

Depressive state was measured using the Inventory of Depressive Symptomatology - Self Report (IDSSR; (32)) to capture changes in symptom severity, and the World Health Organisation's Composite Diagnostic Interview - Short Form (CIDI-SF; (33)) to identify people meeting DSM-5 criteria for MDD at each timepoint. These two measurements were used to identify different operationalisations of depression across follow-up, summarised in supplementary additional file 2. Briefly, participants were categorised as being "symptomatic" (scoring $\geq 26$ on the IDS-SR and meeting CIDI-SF criteria for MDD), having "some symptoms" (scoring $\leq 25$ on the IDS-SR and meeting CIDI-SF criteria for MDD; or > 21 on the IDS-SR and not meeting CIDI-SF criteria for MDD) or having "no symptoms" (scoring $\leq 21$ on the IDS-SR and not meeting CIDI-SF criteria for MDD).

As described previously (22), the primary outcome of interest in RADAR-MDD is depressive relapse, defined here as switching from a state of "no symptoms" to "symptomatic" over a period of 6-months. Secondary depression outcomes are: remission (switching from a state of "symptomatic" to "no symptoms" over a period of 6-months); and change in the severity of depressive symptoms (measured via the continuous IDS-SR).

\section{Anxiety}

Anxiety was measured via the 7-item Generalised Anxiety Disorder questionnaire (GAD7; (34)), used as a continuous indicator of anxiety symptom severity (a total of 21 , with higher scores indicating increased anxiety severity) and a total score $\geq 10$ indicating significant symptoms. This threshold has previously been shown to have good levels of sensitivity and specificity (35).

\section{Functional Ability}

Functional ability was measured using the Work and Social Adjustment Scale (WSAS; (36), using a continuous score from 0-40 to describe the level of impairment, with scores of $0-10,11-20$ and $>20$ to indicate no, some and significant impairment respectively (36). 


\section{Alcohol Use}

The Alcohol Use Disorders Identification Test (AUDIT; (37)) was used to measure alcohol use across timepoints. A total score out of 40 describes the level of alcohol use; scores of 0-7 indicate low risk alcohol consumption; 8-15 indicate hazardous alcohol consumption; 16-19 indicate harmful alcohol consumption; and scores $>20$ indicate likely alcohol dependence. (38)

\section{Illness Perceptions}

The Brief Illness Perceptions Questionnaire (BIPQ; (39)) measured emotional and cognitive representations of illness, capturing perceptions relating to illness identity, causes, control, consequences, timeline, concern, understanding and emotional response. Total scores for each domain can be used individually, or totalled, with higher scores representing a more threatening view of their illness.

\section{Health Service Use}

Access to health services, as well as changes in treatment, and care received was measured via a modified Client Service Receipt Inventory (CSRI; (40)), adapted to be suitable for online delivery and participant self-report.

\section{Covariates}

\section{Life Events}

Any significant life events which may have happened between outcome assessments were measured via the List of Threatening Experiences Questionnaire (LTE-Q; (41)). Changes in employment status were recorded regularly as part of the CSRI (40).

\section{Medication Adherence}

Self-reported adherence to depression medication was measured with the 5-item Medication Adherence Report Scale (MARS-5; (42)).

\section{Statistical Analyses}

Baseline characteristics of the sample were described using means and standard deviations or numbers and percentages as appropriate. To examine whether depressed mood is associated with the availability of data across all modes of data collection, participants were divided using scores on the IDS-SR and CIDI-SF (see additional file 2 for operationalisation) into those who are symptomatic at baseline and those who are not (those with no symptoms and some symptoms are pooled together due to the low number of people with no symptoms at baseline $(\mathrm{N}=4)$ ). Chi-squared tests examined between groupdifferences in categorial data, and linear regressions in continuous data. 
The number and percentage of people who have provided any data via the aRMT and pRMT apps and the wearable device throughout the course of follow-up have been summarised, then divided into quartiles to examine the numbers of people who have provided $0-25 \%$ of expected data, $26-50 \%, 51-75 \%$ and $>75 \%$ of data throughout follow-up. Fitbit wear time estimates were calculated based on the presence of a single heart rate value, greater than zero, per 15-minute window.

P-values comparing the amount of data available between people with symptoms of depression at baseline and those without symptoms of depression at baseline were created using Chi-Squared tests. Ttests compared the number of ESM questions completed in total across all follow-up timepoints between those with and without depression symptoms at baseline. Data were analysed using STATA v16.0.

\section{Results}

\section{Recruitment and Retention Rates}

The first person was enrolled in RADAR-MDD on $30^{\text {th }}$ November 2017, and recruitment ended on $3^{\text {rd }}$ June 2020 , representing a total of 30 months of recruitment. Figure 1 shows the participation rate, detailing the total number of participants contacted and the reasons for non-participation.

Figure 2 shows the participant retention rate throughout the period of follow-up. At each timepoint, the number of people eligible for contact for an outcome assessment decreased as: a) more people had reached the end of the data collection period; and b) as people had been withdrawn from the study. As the last participant was recruited in June 2020 and the study finished in April 2021, the minimum and maximum lengths of possible follow-up were 11 months and 24 months respectively. The completion rate of the primary and secondary outcomes in those who were eligible to complete it (those who had not already completed the study or been previously withdrawn) was approximately $80 \%$ throughout follow-up assessments.

Of the 623 participants enrolled in the study, 445 (71.4\%) provided outcome data at 1-year follow-up and 181 (29.1\%) participated for a full 2-years. A total of 497 people (79.8\%) participated for the maximum possible duration (from their enrolment until the end of data collection in April 2021), and 126 people $(20.2 \%)$ withdrew prematurely. Reasons for withdrawal are provided in additional file 3 . The most common reason for withdrawal across all timepoints was loss to follow-up $(\mathrm{N}=47)$ and problems using the Android study phone (for those who had switched from an iPhone for the purposes of the study; $\mathrm{N}=$ 14), representing $37.3 \%$ and $11.1 \%$ of all withdrawals respectively. A total of 8 participants identified study burden as the main reason for withdrawal, including finding the study "too demanding" $(\mathrm{N}=6)$ or the study "not meeting expectations" $(\mathrm{N}=2)$.

\section{FIGURE 1. Recruitment into RADAR-MDD}

FIGURE 2. Retention of participants throughout follow-up. 
Figure 2 legend: Participants "not contacted" because they had already completed the maximum amount of follow-up time or had already withdrawn from the study. Participants were "contacted" when they were still active participants. *Reasons for withdrawal provided in additional file 3. ${ }^{*}$ Invalid outcomes collected \pm 21 days of due date.

\section{Sample Characteristics}

The target sample size of 600 , across the three sites, was exceeded, with 623 individuals successfully enrolled in the study. The baseline sociodemographic and clinical characteristics of this sample are displayed in Table 1, with comparisons made between those with no/some symptoms at baseline and those who are symptomatic at baseline (see additional file 4 for between-site stratification).

In comparison to those with no/some depression symptoms at baseline, the symptomatic group were: significantly younger; had a higher proportion female, on long-term sick leave or unemployed, receiving any kid of benefit, and earning less than $£ / € 15,000$ per annum. Regarding clinical characteristics, the symptomatic group had a higher proportion of current smokers, medical comorbidities, as well as increased levels of current depression, anxiety, functional disability, and worsened illness perceptions, although lower levels of alcohol use.

\section{Data collection with RMT}

Data collection started on $30^{\text {th }}$ November 2017, with data collection continuing until the last participant was unenrolled from the study on $1^{\text {st }}$ May 2021, resulting in a median study duration of participation of 541 days (interquartile range (IQR): 401-730 days, range: 0-1217 days). In total, data were collected from two aRMT data sources: the RADAR-base aRMT app (PHQ8, RSES and speech tasks); and the THINC-it ${ }^{8}$ app (PDQ5, codebreaker, spotter, symbol check and trails tasks). Data were collected from two pRMT data sources: the Fitbit (heart rate, steps, sleep stages, activity, and calories); and the RADAR-base pRMT app (battery level, gyroscope, ambient noise, ambient light, GPS location, nearby Bluetooth devices, phone usage, and app usage). A total of 2.9 terabytes of compressed data were collected, with $110(17.7 \%)$ participants having more than $50 \%$ available data across all modes of data collection.

\section{Data collected via aRMT}

Figures 3a-c display active RMT data collection stratified baseline depression status. Overall, participants completed a median of 21 (IQR:9-31) PHQ-8 questionnaires, 20 (IQR:9-30) RSES questionnaires, 12 (IQR:2-23) speech tasks. A total of $95.3 \%, 94.5 \%$ and $82.2 \%$ of participants had any data available for the PHQ8, RSES and speech tasks respectively. Chi squared tests found no significant differences in data availability between those with or without depression symptoms at baseline for the PHQ8 $\left(\mathrm{X}^{2}(622\right.$, $N=623)=3.0, p=0.38), \operatorname{RSES}\left(X^{2}(622, N=623)=3.83, p=0.28\right)$, or speech task $\left(X^{2}=4.8, p=0.19\right)$. The 
mean numbers of ESM items completed by those with and without depression symptoms at baseline throughout the study duration were $11.8(S D=23.7)$ and $11.9(S D=23.7)$ respectively, with t-tests demonstrating no significant difference in ESM data availability between these groups $(p=0.07)$.

Figure 4 displays THINC-it app ${ }^{\circledR}$ data collection stratified baseline depression symptom status. Overall, participants completed a median of 5 (IQR:2-10) THINC-it app® PDQ5 questionnaires, 5 (IQR:2-9) Code Breaker tasks, 5 (IQR:2-9) Spotter tasks, 5 (IQR-2-9) Symbol Check tasks, and 5 (IQR=2-10) Trails tasks. A total of $90.5 \%, 84.4 \%, 84.8 \%, 84.6 \%$ and $89.9 \%$ of participants had any data available for the PDQ5, Code Breaker, Spotter, Symbol Check and Trails test respectively. Chi squared tests found no significant differences in data availability between those with or without depression at baseline for the PDQ5 $\left(X^{2}(622, N=623)=2.5, p=0.48\right)$, Code Breaker $\left(X^{2}(622, N=623)=0.91, p=0.82\right)$, Spotter $\left(X^{2}(622, N=623)\right.$ $=1.28, p=0.73)$, Symbol Check $\left(X^{2}(622, N=623)=1.26,0.74\right)$ or Trails $\left(X^{2}(622, N=623)=2.0, p=0.58\right)$ tasks.

\section{FIGURE 3. Illustration of data collected via RADAR-base aRMT app}

Figure 3 legend: Questionnaires triggered every two weeks; maximum number of possible responses: 52. 3a: 8-item Patient Health Questionnaire (PHQ8); 3b: Rosenberg Self-Esteem Scale (RSES); 3c: Speech data.

\section{FIGURE 4. Illustration of data collected via THINC-it app}

Figure 4 legend: Questionnaires triggered every 6 weeks; maximum number of possible responses: 17. 4a: 5-item Perceived Deficits Questionnaire (PDQ5); 4b: Code Breaker; 4c: Spotter; 4d: Symbol Check; 4e: Trails.

\section{Data collected via wearable technology}

Table 2 displays wearable RMT data collection using Fitbit, stratified by baseline depression status. Data collection relied on 1) participants wearing the Fitbit device, 2) regularly charging and syncing the Fitbit device; 3 ) data being returned/provided by the Fitbit servers. Fitbit wear-time varied during the study (Figure 5a), with the average participant wear-time across the entire duration of follow-up estimated as 62.5\% (SD: 9.1 percentage points; Figure 5b), and the average number of hours per day as 15.1 hours (SD: 2.2 hours). Wear-time decreased over time and wear-time did not significantly differ between those with no depression symptoms versus those with symptoms at baseline $\left(X^{2}(622, N=623)=525616\right.$, $p=0.24)$.

Step count data were the most frequently available data, with almost $50 \%$ of participants providing $>75 \%$ of expected data throughout the course of follow-up. Activity data (comprising a combination of data derived from Fitbit proprietary algorithms and via participants inputting their own activities manually) was the least readily available data, with only $5 \%$ of participants having $>75 \%$ data availability. Activity data are also the only data type found to have significantly different levels of availability according to the 
presence of depression at baseline or $\operatorname{not}\left(X^{2}(622, N=623)=14.1, p=0.002\right)$. In comparison to those without depression at baseline, those identified as symptomatic at baseline had a significantly larger percentage of people providing $<26 \%$ of activity data, and smaller percentage of people providing $>25 \%$ of data throughout the course of follow-up. Figure 5a shows a paler horizontal band of colour between days 290 and 380 of study participation, indicating lower levels of wear-time during these time-points. and figure $5 \mathrm{~b}$ shows a dip in percentage wear-time in people with symptoms of depression at baseline after the first year of participation.

\section{Data collected via PRMT}

Table 3 displays passive data collection across all smartphone sensors, stratified by the presence of baseline depression. The most data were available for GPS location and battery level data. The least data were available for phone usage. No evidence of a difference in data availability between those with and without depression at baseline was identified.

\section{FIGURE 5. Illustration of Fitbit data streams.}

Figure 5 legend: A) Heatmap representing study day and data points per hour. B) percentage wear time stratified by baseline depression status.

\section{Discussion}

\section{Study Recruitment and Retention}

Recruitment into RADAR-MDD was highly successful, with the flexibility of face-to-face and remote enrolments resulting in the study exceeding its recruitment targets despite the COVID-19 pandemic (43). Attrition rates in longitudinal research vary widely (44) and whilst there is no recognised threshold for "acceptable" versus "unacceptable" dropout, follow-up levels of $50 \%, 60 \%$ and $70 \%$ have previously been described as adequate, good and very good respectively (45). We report $~ 80 \%$ completion rates of our outcomes across all follow-up timepoints, with $79.8 \%$ of all enrolled individuals completing the study protocol for the maximum amount of time possible, representing excellent availability of our primary and secondary outcome measures.

\section{Sociodemographic Characteristics}

The RADAR-MDD cohort has a higher proportion of White and Female individuals than would typically be seen in the general population or depressed population (46), reflecting the tendency for White Females to attend mental health services more often than their male/non-White counterparts, and their greater likelihood of participating in research studies $(47,48)$, however Ethnicity data were only collected in London and Amsterdam. The mean age and gender distribution in our participants is comparable to other 
MDD samples, such as Sequenced Treatment Alternatives to Relieve Depression (STAR*D; (49)) and the Rhode Island Methods to Improve Diagnostic Assessment and Services (MIDAS; (50)). These characteristics may limit the generalisability of our findings to the wider population. It is also worth noting that ethnic groups across the two countries who collected ethnicity data are challenging to compare, meaning that in-depth interrogation of racial differences in outcomes will be affected by small cell sizes unless ethnic groups are merged into larger, less descriptive categories. In terms of clinical presentation, our sample have slightly lower levels of current depression severity and reduced WSAS functional disability than those recruited into STAR*D (49).

\section{RMT Domains and Data Availability}

Data availability varied across the RMT domains. Over $90 \%$ of participants had data available for analysis from the aRMT, with the PHQ-8 and RSES having the largest amount of data available for the most people. The least amount of aRMT data was available for all assessments conducted via the THINC-it ${ }^{\circledR}$ app, with $<26 \%$ of expected data available in approximately $60 \%$ of participants. There are several explanations for this difference in data availability in comparison to our other aRMT assessments. Firstly, due to the technical requirements of integrating data from the separate THINC-it ${ }^{\circledR}$ app into the RADAR-base platform, the first THINC-it@ data were received in March 2018, with the first 4months of data collection excluding THINC-it ${ }^{\circledR}$ data. There were also initial challenges syncing data collected via the THINC-it with the RADAR-bae platform, meaning there was potential for data loss in the early months of data collection. Secondly, the THINC-it@ app is separate to the other RADAR-base apps, with different branding, design and feel to the RADAR-base apps. This may have made the tasks appear separate or "other" to the main protocol and reduced adherence to these tasks. The THINC-it $\circledast$ app does not have an inbuilt notification system; participants received notifications to complete the cognitive tasks via the RADAR-base aRMT app. Participants are required to switch between apps, which increases the number of points at which interest or motivation may be lost (51). Finally, the cognitive tasks offered as part of the THINC-it can require more attention than conventional questionnaires which may be more challenging for those who are experiencing depression symptoms.

We report an overall Fitbit wear-time of $62.5 \%$, across a median study participation of 541 days, and a mean wear-time of 15.1 hours per day. This is lower than the wear-time of 22.6 hours per day across a two-year follow-up period in a recent United States population-based Fitbit study (52). However, this study omitted missing wear time data, and excluded measurements with a wear-time lower than 1000 minutes per day which inflates their wear-time statistics. In contrast to our sample, this study (52) used a nonclinical population and the barriers to long-term use of a wearable device are likely to be different in an MDD versus general population sample (18). Comparatively, Pedrelli et al., 2020 report Empatica E4 weartime estimates of $92-94 \%$ in their study involving 31 individuals with MDD, however their follow-up period was limited to 8-weeks (53). Although similar in clinical characteristics, our length of follow-up and integration of a wearable into a more complex set of data collection sources limit meaningful comparisons from being made.

Page $15 / 33$ 
To the best of our knowledge, no remote measurement studies have reported the quantity of data collected via smartphone sensors. The largest amount of data were available for battery level and GPS sensors. For a multiparametric analysis, data across multiple sensor types will be needed. We report a total of 110 individuals ( $17.7 \%$ of the entire sample) who have $>50 \%$ of data for data types. It is important to acknowledge this as an indicator of the amount of resource and data collection required for multiparametric analyses. Although a remote study by nature, participants had close contact with the research team throughout the study; the researchers were available for technical support and questionnaire reminders, in addition to conducting risk assessments based on questionnaire answers. Future work will need to investigate the minimum amount of contact time required to acquire usable data, for real-world implementation to be viable.

\section{Limitations}

There are several limitations and challenges presented by the current paper. Firstly, each of the sensor and data types collected has different temporal validity and aggregation requirements. For example, sleep data are only meaningful when aggregated from midday-midday, whereas activity data are more relevant when calculated from midnight-midnight. At a granular level, data from smartphone and wearable sensors are so fine that no meaningful inferences can be gained, requiring some form of aggregation which may not be the same across different sensors; whereas heart-rate data might be collected every 5 seconds and summarised across an hour, the aggregation of GPS data is dependent on the smartphone device being used. In the current paper we have endeavoured to summarise data availability as coherently as possible within these constraints, aiming to provide an easily replicable, comparable, and interpretable description of the data available within our dataset.

It is also essential to acknowledge the technical challenges inherent to multimodal data collection across long periods of time. RADAR-base and its associated apps were developed and piloted within the main data collection period, with iterative changes and updates being made throughout the course of followup. These changes may have been implemented to overcome a system-related issue introduced by the updates to the Android operating system, or in direct response to participant or researcher feedback. This flexibility in app design and development is essential to maintain app compatibility; this means that an individual participating throughout 2019-2020 will have had a different user experience to an individual participating throughout 2020-2021.

Whilst the majority of our recruitment occurred before the global pandemic, the threat posed by COVID-19 may have affected existing participants' research experience; recent evidence suggests that people with moderate/severe levels of depression who are already enrolled in a research study show a reduced ability and desire to adhere to research protocols due to COVID-19 (54). Given the high level of depressive symptoms in our sample, the pandemic and its associated social interventions may have added a burden to participants resulting in an increased dropout rate and reduced adherence to the study protocol. We have previously reported the impact of the pandemic and associated social interventions on the data 
collected via RMT across the RADAR-CNS clinical studies (55) and future work will extend this to examine how the pandemic may have affected data availability.

Despite these limitations, RADAR-MDD remains the largest, most ambitious multimodal RMT study in the field of mental health. A recent systematic review summarising studies using passive and active smartphone-based measurements in affective disorders found only 5 studies in people with MDD, and these studies reported a median sample size of 5 , and median follow-up time of 4 weeks (56). It is essential to remember the scope of the RADAR-MDD research protocol and to be mindful of the exploratory nature of this novel method of data collection. This paper is the first to describe an extremely complex set of data as transparently as possible, to clarify the extent of the data available for future analyses.

\section{Future Research}

There are some vital next steps in the exploration of RADAR-MDD data which will be examined in addition to the primary objectives of the RADAR-MDD study (22). Firstly, as reported earlier, the present paper reports the amount of data available across all modes of data collection. A more thorough investigation into the quality of the data is warranted before more complex analyses are conducted. Furthermore, whilst we have provided some preliminary results indicating no evidence of a link between baseline depression status and data availability, a much more detailed and rigorous investigation is needed. It is likely that fluctuations in depression symptoms over time are more relevant for predicting technology use, rather than a static baseline status and we will be examining this in more detail in the future. Our next steps will also be to examine sociodemographic, clinical and technical predictors of data availability; a separate paper dedicated to this question is needed to do justice to the amount of data available and the importance of understanding missing data in this context.

\section{Conclusion}

The data collected in RADAR-MDD indicates that collecting RMT data from clinical populations is feasible. We found comparable levels of data availability in active (requiring input from the participant) and passive (requiring no input from the participant) forms of data collection, demonstrating that both are feasible in this patient group. However, data availability will depend on the data type, with higher burden data sources (such as cognitive tasks, or keeping wearable devices charged) reducing data availability. There was no convincing indication that the severity of depression symptoms at baseline was associated with data availability, in this sample. The next steps are to illustrate the predictive value of these data, which will be the focus of our future data analysis aims.

\section{Declarations}

\section{Ethical Approvals and Consent to Participate}


Ethical approvals for study conduct were obtained from the Camberwell St Giles Research Ethics Committee (REC reference: 17/LO/1154), in London from the CEIC Fundacio Sant Joan de Deu (Cl: PIC128-17) in Barcelona, and from the Medische Ethische Toetsingscommissie VUms (METc VUmc registratienummer: 2018.012 - NL63557.029.17) in the Netherlands. RADAR-MDD was conducted per the Declaration of Helsinki and Good Clinical Practice, adhering to principles outlined in the NHS Research Governance Framework for Health and Social Care (2nd edition).

\section{Consent to Publish}

Not applicable

\section{Availability of Data and Materials}

The datasets used and/or analysed during the current study are available from the corresponding author on reasonable request.

\section{Competing Interests}

JCB and PA are full-time employees of $\mathrm{H}$. Lundbeck A/S. DCM has accepted honoraria and consulting fees from Apple, Inc., Otsuka Pharmaceuticals, Pear Therapeutics, and the One Mind Foundation, royalties from Oxford Press, and has an ownership interest in Adaptive Health, Inc. NVM is an employee of Janssen Pharmaceutica NV and may hold company equity.

QL, NM, SV and VN are employees of Janssen Research \& Development, LLC and hold company stocks/stock options. JMH has received economic compensation for participating in advisory boards or giving educational lectures from Eli Lilly \& Co, Sanofi, Lundbeck, and Otsuka. No other authors have competing interests to declare.

\section{Funding}

The RADAR-CNS project has received funding from the Innovative Medicines Initiative 2 Joint Undertaking under grant agreement No 115902. This Joint Undertaking receives support from the European Union's Horizon 2020 research and innovation programme and EFPIA (www.imi.europa.eu). This communication reflects the views of the RADAR-CNS consortium and neither IMI nor the European Union and EFPIA are liable for any use that may be made of the information contained herein. The funding body have not been involved in the design of the study, the collection or analysis of data, or the interpretation of data. 


\section{Author's contributions}

FM has contributed to the design and coordination of the study in London, as well as leading on data analysis and write-up of this manuscript. DL has contributed to data processing and analysis. SS has contributed to design and coordination of the study in Barcelona. FL has contributed to design and coordination of the study in Amsterdam. KMW has contributed to the study conduct in London. PA has contributed to the design and development of the study. GG has contributed to the design and development of the study. SD has contributed to the study conduct in Amsterdam. JMH has contributed to the study design and conduct in Barcelona. MHorsfall has contributed to the study conduct in Amsterdam. Al has contributed to the study conduct in London. GL has contributed to the study conduct in London. QL has contributed to the development and design of the study. FL has contributed to the study conduct in Barcelona. DCM has contributed to the design and development of the study. VN has contributed to the design and development of the study. CO has contributed to the study conduct in London. BWJHP has contributed to the development and design of the study. SS has contributed to the development and design of the study. TW has contributed to the development and design of the study. SB has contributed to the design and development of the study. CB has contributed to the design and development of the study. IMG has contributed to the design and development of the study. AR has contributed to the design and development of the study. PC has contributed to the development of the RADAR-base system used for data collection and management across all sites, data protection, security and storage systems. RJD, AF, YR, ZR and CS have contributed to the development of the RADAR-base system used for data collection and management across all sites, data protection, security and storage systems. NC has contributed to data processing and interpretation. NVM has contributed to data processing and interpretation. SV has contributed to data processing and interpretation. MHotopf has contributed to study development and design and conduct.

All authors have all been involved in reviewing the manuscript and have given approval for the it to be published. All authors have agreed to be accountable for all aspects of the work, ensuring that questions relating to the accuracy or the integrity of any part of the work are appropriately investigated and resolved.

\section{Acknowledgements}

This paper represents independent research part funded by the National Institute for Health Research (NIHR) Maudsley Biomedical Research Centre at South London and Maudsley NHS Foundation Trust and King's College London. The views expressed are those of the author(s) and not necessarily those of the NHS, the NIHR or the Department of Health and Social Care. We thank all the members of the RADAR-CNS patient advisory board for their contribution to the device selection procedures, and their invaluable advice throughout the study protocol design. We also thank Line Kühnel for providing feedback on an early version of this manuscript. Participant recruitment in Amsterdam was partially accomplished through Hersenonderzoek.nl, a Dutch online registry that facilitates participant recruitment for 
neuroscience studies (https://hersenonderzoek.nl/). Hersenonderzoek.nl is funded by ZonMw-Memorabel (project no 73305095003), a project in the context of the Dutch Deltaplan Dementie, Gieskes-Strijbis Foundation, the Alzheimer's Society in the Netherlands and Brain Foundation Netherlands. Participants in Spain were recruited through the following institutions: Parc Sanitari Sant Joan de Déu network of mental health services (Barcelona); Institut Català de la Salut primary care services (Barcelona); Institut Pere Mata-Mental Health Care (Tarrassa); Hospital Clínico San Carlos (Madrid).

This research was reviewed by a team with experience of mental health problems and their carers who have been specially trained to advise on research proposals and documentation through the Feasibility and Acceptability Support Team for Researchers (FAST-R): a free, confidential service in England provided by the National Institute for Health Research Maudsley Biomedical Research Centre via King's College London and South London and Maudsley NHS Foundation Trust.

RJBD is supported by the following: (1) NIHR Biomedical Research Centre at South London and Maudsley NHS Foundation Trust and King's College London, London, UK; (2) Health Data Research UK, which is funded by the UK Medical Research Council, Engineering and Physical Sciences Research Council, Economic and Social Research Council, Department of Health and Social Care (England), Chief Scientist Office of the Scottish Government Health and Social Care Directorates, Health and Social Care Research and Development Division (Welsh Government), Public Health Agency (Northern Ireland), British Heart Foundation and Wellcome Trust; (3) The BigData@Heart Consortium, funded by the Innovative Medicines Initiative-2 Joint Undertaking under grant agreement No. 116074. This Joint Undertaking receives support from the European Union's Horizon 2020 research and innovation programme and EFPIA; it is chaired by DE Grobbee and SD Anker, partnering with 20 academic and industry partners and ESC; (4) the National Institute for Health Research University College London Hospitals Biomedical Research Centre; (5) the National Institute for Health Research (NIHR) Biomedical Research Centre at South London and Maudsley NHS Foundation Trust and King's College London; (6) the UK Research and Innovation London Medical Imaging \& Artificial Intelligence Centre for Value Based Healthcare; (7) the National Institute for Health Research (NIHR) Applied Research Collaboration South London (NIHR ARC South London) at King's College Hospital NHS Foundation Trust.

\section{References}

1. James SL, Abate D, Abate KH, Abay SM, Abbafati C, Abbasi N, et al. Global, regional, and national incidence, prevalence, and years lived with disability for 354 diseases and injuries for 195 countries and territories, 1990-2017: A systematic analysis for the Global Burden of Diseases Study 2017. Lancet. 2018;392:1789-858. 
2. Beratis S, Katrivanou A, Georgiou S, Monastirli A, Pasmatzi E, Gourzis P, et al. Major depression and risk of depressive symptomatology associated with short-term and low-dose interferon- a treatment. J Psychosom Res. 2005;doi: 10.1016/j.jpsychores.2004.03.010.

3. Cambridge OR, Knight MJ, Mills N, Baune BT. The clinical relationship between cognitive impairment and psychosocial functioning in major depressive disorder: A systematic review. Psychiatr Res. 2018; doi: 10.1016/j.psychres.2018.08.033.

4. Saragoussi D, Christensen MC, Hammer-Helmich L, Rive B, Touya M, Haro JM. Long-term follow-up on health-related quality of life in major depressive disorder: A 2-year european cohort study. Neuropsychiatr Dis Treat. 2018;14:1339-50.

5. Miloyan B, Fried E. A reassessment of the relationship between depression and all-cause mortality in 3,604,005 participants from 293 studies. World Psychiatr. 2017; doi: 10.1002/wps.20439.

6. Verduijn J, Verhoeven JE, Milaneschi Y, Schoevers RA, van Hemert AM, Beekman ATF, et al. Reconsidering the prognosis of major depressive disorder across diagnostic boundaries: full recovery is the exception rather than the rule. BMC Med. 2017; doi: 10.1186/s12916-017-0972-8.

7. Blanco C, Okunda M, Markowitz, John C, Liu S-M, Grant, Bridget F, Hasin, Deborah S. The epidemiology of chronic major depressive disorder and dysthymic disorder: Results from the National Epidemiologic Survey on Alcohol and Related Conditions. J Clin Psychiatr. 2010;17:164556.

8. Wells JE, Horwood LJ. How accurate is recall of key symptoms of depression? A comparison of recall and longitudinal reports. Psychol Med. 2004;34:1001-11.

9. Mohr DC, Shilton K, Hotopf M. Digital phenotyping, behavioral sensing, or personal sensing: names and transparency in the digital age. npj Dig Med. 2020: doi: 10.1038/s41746-020-0251-5.

10. Marzano L, Hollis C, Cipriani A, Malhi GS. Digital technology: Coming of age? Ev Based Mental Health. 2017;20:97.

11. Cornet VP, Holden RJ. Systematic review of smartphone-based passive sensing for health and wellbeing. J Biomen Inform. 2018. Doi: 10.1016/j.jbi.2017.12.008.

12. Myin-Germeys I, Oorschot M, Collip D, Lataster J, Delespaul P, van Os J. Experience sampling research in psychopathology: opening the black box of daily life. Psychol Med. 2009;39:1533-47.

13. Firth J, Torous J, Nicholas J, Carney R, Pratap A, Rosenbaum S, et al. The efficacy of smartphonebased mental health interventions for depressive symptoms: a meta-analysis of randomized controlled trials. World Psychiatry. 2017. 16:287-98.

14. Digital Health Funding and M\&A 2018 Fourth Quarter and Annual Report. 2018. Available from: http://www.mercomcapital.com.

15. Localytics. App Uninstall Statistics 2019: 25\% App Abandonment. 2019. Available from:https://uplandsoftware.com/localytics/resources/blog/25-of-users-abandon-apps-after-oneuse/.

16. Fleming T, Bavin L, Lucassen M, Stasiak K, Hopkins S, Merry S. Beyond the trial: Systematic review of real-world uptake and engagement with digital self-help interventions for depression, low mood, or 
anxiety. J Med Internet Res. 2018. 20:e199.

17. Greer B, Robotham D, Simblett S, Curtis H, Griffiths H, Wykes T. Digital exclusion among mental health service users: a qualitative investigation. J Med Internet Res. 2019. Doi: 10.2196/11696.

18. Simblett S, Matcham F, Siddi S, Bulgari V, di San Pietro CB, López JH, et al. Barriers to and facilitators of engagement with mHealth technology for remote measurement and management of depression: Qualitative analysis. JMIR mHealth uHealth. 2019. Doi: 10.2196/11325.

19. Szinay D, Jones A, Chadborn T, Brown J, Naughton F. Influences on the uptake of and engagement with health and well-being smartphone apps: Systematic review. JMIR. 2020. Doi: 10.2196/17572.

20. Pham Q, Graham G, Carrion C, Morita PP, Seto E, Stinson JN, et al. A library of analytic indicators to evaluate effective engagement with consumer mHealth apps for chronic conditions: Scoping review. JMIR mHealth uHealth. 2019. 7;e11941.

21. Remote Assessment of Disease and Relapse - Central Nervous System. RADAR-CNS. 2018. Available from: www.radar-cns.org.

22. Matcham F, Barattieri Di San Pietro C, Bulgari V, De Girolamo G, Dobson R, Eriksson H, et al. Remote assessment of disease and relapse in major depressive disorder (RADAR-MDD): A multi-centre prospective cohort study protocol. BMC Psychiatry. 2019; doi: 10.1186/s12888-019-2049-z.

23. Simblett S, Greer B, Matcham F, Curtis H, Polhemus A, Ferrão J, et al. Barriers to and facilitators of engagement with remote measurement technology for managing health: Systematic review and content analysis of findings. JMIR. 2018. 20:210480.

24. Bot M, Middeldorp CM, de Geus EJC, Lau HM, Sinke M, van Nieuwenhuizen B, et al. Validity of LIDAS (LIfetime Depression Assessment Self-report): a self-report online assessment of lifetime major depressive disorder. Psychol Med. 2017. 47:279-89.

25. Ranjan Y, Rashid Z, Stewart C, Conde P, Begale M, Verbeeck D, et al. RADAR-base: Open source mobile health platform for collecting, monitoring, and analyzing data using sensors, wearables, and mobile devices. JMIR mHealth uHealth. 2019. Doi: 10.2196/11734.

26. Kroenke K, Strine TW, Spitzer, Robert L, Williams J, Berry JT, Mokdad AH. The PHQ-8 as a measure of current depression in the general population. J Affect Disord. 2009.114:163-73.

27. Greenberger E, Chen C, Dmitrieva J, Farruggia SP. Item-wording and the dimensionality of the Rosenberg Self-Esteem Scale: do they matter? Pers Individ Dif 2003. 35:1241-54.

28. Williamson JR, Quatieri TF, Helfer BS, Ciccarellu G, Mehta DD. Vocal and facial biomarkers of dperession based on motor incoordination and timing. In: 4th International Audio/Visual Emotion Challenge and Workshop: Depression Challenge. 2014.doi: 10.1145/2661806.2661809.

29. Mclntyre RS, Best MW, Bowie CR, Carmona NE, Cha DS, Lee Y, et al. The THINC-integrated tool (THINC-it) screening assessment for cognitive dysfunction: Validation in patients with major depressive disorder. J Clin Psychiatry. 2017. 78:873-81.

30. Lam RW, Lamy FX, Danchenko N, Yarlas A, White MK, Rive B, et al. Psychometric validation of the perceived deficits questionnaire-depression (PDQ-D) instrument in US and UK respondents with major depressive disorder. Neuropsychiatr Dis Treat. 2018. 14:2861-77. 
31. Harris PA, Taylor R, Thielke R, Payne J, Gonzalez N, Conde JG. Research electronic data capture (REDCap)-A metadata-driven methodology and workflow process for providing translational research informatics support. J Biomed Inform. 2009. Doi: 10.1016/j.jbi.2008.08.010

32. Rush AJ, Carmody T, Reimitz P-E. The Inventory of Depressive Symptomatology (IDS): Clinician (IDSC) and Self-Report (IDS-SR) ratings of depressive symptoms. Int J Methods Psychiatr Res. 2000. 9:45-59.

33. Kessler RC, Andrews G, Mroczek D, Ustun B, Wittchen H-U. The World Health Organization Composite International Diagnostic Interview short-form (CIDI-SF). Int J Methods Psychiatr Res. 1998. 7:171-85.

34. Spitzer RL, Kroenke K, Williams JBW, Löwe B. A brief measure for assessing generalized anxiety disorder: the GAD-7. Arch Intern Med. 2006. 166: 1092-7.

35. Jordan P, Shedden-Mora MC, Löwe B. Psychometric analysis of the Generalized Anxiety Disorder scale (GAD-7) in primary care using modern item response theory. PLoS One. 2017.

Doi: $10.1371 /$ journal.pone.0182162

36. Mundt JC. The Work and Social Adjustment Scale: a simple measure of impairment in functioning. Br J Psychiatry. 2002. 180;461-4.

37. Daeppen J-B, Yersin B, Landry U, Pecoud A, Decrey H. Reliability and Validity of the Alcohol Use Disorders Identification Test (AUDIT) Imbedded within a general health risk screening questionnaire: Results of a survey in 332 primary care patients. Alcohol Clin Exp Res. 2000. Doi: 10.1111/j.15300277.2000.tb02037.x

38. Babor TF, Robaina K. The Alcohol Use Disorders Identification Test (AUDIT): A review of graded severity algorithms and national adaptations. Int J Alcohol Drug Res. 2016. 5:17-24

39. Broadbent E, Petrie KJ, Main J, Weinman J. The brief illness perception questionnaire. J Psychosom Res. 2006. 60;631-7.

40. Chisholm D, Knapp MRJ, Knudsen HC, Amaddeo F, Gaite L, Van Wijngaarden B. Client SocioDemographic and Service Receipt Inventory - European Version: development of an instrument for international research. Br J Psychiatry. Doi: 10.1192/bjp.177.39.s28

41. Brugha TS, Cragg D. The List of Threatening Experiences: the reliability and validity of a brief life events questionnaire. Acta Psychiatr Scand. 1990. Doi: 10.1111/j.1600-0447.1990.tb01360.

42. Chan AHY, Horne R, Hankins M, Chisari C. The Medication Adherence Report Scale: A measurement tool for eliciting patients' reports of nonadherence. Br J Clin Pharmacol. 2020. 86;1281-8.

43. Oetzmann C, White KM, Ivan A, Julie J, Leightley D, Lavelle G, et al. A framework for recruiting into a remote measurement technologies (RMTs) study: Experiences from a major depressive disorder cohort. OSF Preprint. Doi: 10.31219/osf.io/ns7dc.

44. Gustavson K, Von Soest T, Karevold E, Roysamb E. Attrition and generalizability in longitudinal studies: Findings from a 15-year population-based study and a Monte Carlo simulation study. BMC Public Health. 2012. Doi: 10.1186/1471-2458-12-918.

45. Kristman V, Manno M, Côté P. Loss to follow-up in cohort studies: How much is too much? Eur J Epidemiol. 2004. 28;2272-81. 
46. Cardel MI, Manasse S, Krukowski RA, Ross K, Shakour R, Miller DR, et al. COVID-19 impacts mental health outcomes and ability/desire to participate in research among current research participants. Obesity. 2020. Doi: 10.1002/oby.23016

47. Sun S, Folarin AA, Ranjan Y, Rashid Z, Conde P, Stewart C, et al. Using smartphones and wearable devices to monitor behavioral changes during COVID-19. JMIR. 2020. 22;e19992.

48. Arias de la Torre J, Vilagut G, Ronaldson A, Dregan A, Ricci-Cabello I, Hatch SL, et al. Prevalence and age patterns of depression in the United Kingdom. A population-based study. J Affect Disord. 2021. Doi: 10.1016/j.jad.2020.09.129.

49. Manuel Jl. Racial/ethnic and gender disparities in health care use and access. Health Serv Res. 2018. Doi: $10.1111 / 1475-6773.12705$.

50. Brown G, Marshall M, Bower P, Woodham A, Waheed W. Barriers to recruiting ethnic minorities to mental health research: A systematic review. Int J Methods Psychiatr Res. 2014. Doi: 10.1002/mpr.1434.

51. Trivedi MH, John Rush A, Wisniewski SR, Nierenberg AA, Warden D, Louise Ritz M, et al. Evaluation of outcomes with Citalopram for depression using measurement-based care in STAR*D: Implications for clinical practice. Am J Psychiatry. 2006. 163:28-40.

52. Zimmerman M. A review of 20 years of research on overdiagnosis and underdiagnosis in the Rhode Island Methods to Improve Diagnostic Assessment and Services (MIDAS) project. Canadian J Psychiatr. 2016. Doi: 10.1177/0706743715625935.

53. Dahl AJ, D'Alessandro AM, Peltier JW, Swan EL. Differential effects of omni-channel touchpoints and digital behaviors on digital natives' social cause engagement. J Res Interact Mark. 2018. 12:258-73.

54. Radin JM, Wineinger NE, Topol EJ, Steinhubl SR. Harnessing wearable device data to improve statelevel real-time surveillance of influenza-like illness in the USA: a population-based study. Lancet Digit Heal. 2020. 11:e85-93.

55. Pedrelli P, Fedor S, Ghandeharioun A, Howe E, lonescu DF, Bhathena D, et al. Monitoring changes in depression severity using wearable and mobile sensors. Front Psychiatry. 2020. 11:584711.

56. Dogan E, Sander C, Wagner X, Hegerl U, Kohls E. Smartphone-based monitoring of objective and subjective data in affective disorders: Where are we and where are we going? Systematic review. JMIR. 2017. 19:e262.

\section{Tables 2 And 3}

Table 2: Wearable remote measurement technology data availability stratified by baseline depression status. 


\begin{tabular}{|c|c|c|c|c|c|}
\hline $\begin{array}{l}\text { Data } \\
\text { Type }\end{array}$ & $\begin{array}{l}\text { Data } \\
\text { Completion* }\end{array}$ & $\begin{array}{l}\text { Total } \\
\text { Sample } \\
(n=623)\end{array}$ & $\begin{array}{l}\text { No/Some Baseline } \\
\text { Depression } \\
\text { Symptoms } \\
(\mathrm{n}=245)\end{array}$ & $\begin{array}{l}\text { High Baseline } \\
\text { Depression } \\
\text { Symptoms } \\
(\mathrm{n}=378)\end{array}$ & $\begin{array}{l}X^{2} \\
\text { (Pvalue) }\end{array}$ \\
\hline & & N (\%) & N (\%) & $\mathrm{N}(\%)$ & \\
\hline \multirow{6}{*}{$\begin{array}{l}\text { Heart } \\
\text { Rate }\end{array}$} & Any data & $588(94.4)$ & $229(93.4)$ & $359(95.0)$ & \\
\hline & No data & $35(5.6)$ & $16(6.5)$ & $19(5.0)$ & \\
\hline & $0-25 \%$ & $103(16.5)$ & $30(12.2)$ & 73 (19.3) & $\begin{array}{l}5.7 \\
(0.13)\end{array}$ \\
\hline & $26-50 \%$ & 83 (13.3) & $32(13.1)$ & $51(13.5)$ & \\
\hline & $51-75 \%$ & $111(17.8)$ & 47 (19.2) & 64 (16.9) & \\
\hline & $76+\%$ & $326(52.2)$ & $136(55.1)$ & $190(50.6)$ & \\
\hline \multirow[t]{6}{*}{ Steps } & Any data & $587(94.2)$ & $229(93.4)$ & $358(94.7)$ & \\
\hline & No data & $36(6.8)$ & $16(6.6)$ & $20(5.3)$ & \\
\hline & $0-25 \%$ & 120 (19.3) & $38(15.5)$ & $82(21.7)$ & \multirow{5}{*}{$\begin{array}{l}6.5 \\
(0.09)\end{array}$} \\
\hline & $26-50 \%$ & $88(114.1)$ & $30(12.2)$ & $58(15.3)$ & \\
\hline & $51-75 \%$ & 116 (18.6) & $46(18.8)$ & 70 (18.5) & \\
\hline & $76+\%$ & $299(48.0)$ & $131(53.5)$ & $168(44.4)$ & \\
\hline \multirow{6}{*}{$\begin{array}{l}\text { Sleep } \\
\text { (Classic) }\end{array}$} & Any data & $543(87.2)$ & 214 (87.3) & $329(87.0)$ & \\
\hline & No data & $80(12.8)$ & $31(12.7)$ & 49 (13.0) & \\
\hline & $0-25 \%$ & 485 (77.8) & $200(81.6)$ & $285(75.3)$ & $\begin{array}{l}3.9 \\
(0.28)\end{array}$ \\
\hline & $26-50 \%$ & 98 (15.7) & 33 (13.5) & 65 (17.2) & \\
\hline & $51-75 \%$ & $38(6.1)$ & $11(4.5)$ & $27(7.1)$ & \\
\hline & $76+\%$ & $2(0.3)$ & $1(0.4)$ & $1(0.3)$ & \\
\hline \multirow{6}{*}{$\begin{array}{l}\text { Sleep } \\
\text { Stages }\end{array}$} & Any data & $536(86.0)$ & $213(86.9)$ & $323(85.4)$ & \\
\hline & No data & $87(140)$ & $32(13.1)$ & 55 (14.6) & \\
\hline & $0-25 \%$ & $212(34.0)$ & $80(32.7)$ & $132(34.9)$ & $\begin{array}{l}2.3 \\
(0.52)\end{array}$ \\
\hline & $26-50 \%$ & $134(21.5)$ & $58(23.7)$ & $76(20.1)$ & \\
\hline & $51-75 \%$ & $152(24.4)$ & $63(25.7)$ & $89(23.5)$ & \\
\hline & $76+\%$ & $125(20.1)$ & $44(18.0)$ & $81(21.4)$ & \\
\hline
\end{tabular}




\begin{tabular}{|c|c|c|c|c|c|}
\hline \multirow[t]{6}{*}{ Activity** } & Any data & $580(93.1)$ & $226(92.2)$ & 354 (93.7) & \\
\hline & No data & $43(6.9)$ & $19(7.8)$ & $24(6.3)$ & \\
\hline & $0-25 \%$ & $358(57.5)$ & $126(51.4)$ & 232 (61.4) & $\begin{array}{l}14.1 \\
(0.002)\end{array}$ \\
\hline & $26-50 \%$ & $143(23.0)$ & 53 (51.6) & $90(23.8)$ & \\
\hline & $51-75 \%$ & $88(14.1)$ & 48 (19.6) & 40 (10.6) & \\
\hline & $76+\%$ & $34(5.5)$ & $18(9.4)$ & $16(4.2)$ & \\
\hline \multirow[t]{6}{*}{ Calories** } & Any data & $580(93.1)$ & 224 (91.4) & $356(94.2)$ & \\
\hline & No data & $43(6.9)$ & $21(8.6)$ & $22(5.8)$ & $\begin{array}{l}4.57 \\
(0.21)\end{array}$ \\
\hline & $0-25 \%$ & $122(19.6)$ & $40(16.3)$ & $82(21.7)$ & \\
\hline & $26-50 \%$ & 87 (19.6) & 31 (12.7) & $56(14.8)$ & \\
\hline & $51-75 \%$ & $113(18.1)$ & $44(18.0)$ & 69 (18.3) & \\
\hline & $76+\%$ & 301 (48.3) & $130(53.1)$ & $171(45.2)$ & \\
\hline
\end{tabular}

*Calculated as the total number of days in which at least one data point has been provided. ${ }^{*}$ Data collected either via manual input about food/liquid intake from participant, or Fitbit automation from step count data (not possible to delineate source of data).

Table 3: Passive remote measurement technology data availability stratified by baseline depression status and measurement. 


\begin{tabular}{|c|c|c|c|c|c|}
\hline Data Type & $\begin{array}{l}\text { Data } \\
\text { Completion* }\end{array}$ & $\begin{array}{l}\text { Total } \\
\text { Sample } \\
(n=623)\end{array}$ & $\begin{array}{l}\text { No/Some Baseline } \\
\text { Depression } \\
\text { Symptoms } \\
(n=245)\end{array}$ & $\begin{array}{l}\text { High Baseline } \\
\text { Depression } \\
\text { Symptoms } \\
(n=378)\end{array}$ & $\begin{array}{l}X^{2} \\
(P \text { value })\end{array}$ \\
\hline & & $\mathrm{N}(\%)$ & N (\%) & $\mathrm{N}(\%)$ & \\
\hline \multirow[t]{6}{*}{ Battery Level } & Any data & $\begin{array}{l}603 \\
(96.8)\end{array}$ & 232 (94.7) & 371 (98.1) & \\
\hline & No data & $20(3.2)$ & $13(5.3)$ & $7(1.9)$ & \multirow{5}{*}{$\begin{array}{l}4.9 \\
(0.18)\end{array}$} \\
\hline & $0-25 \%$ & $\begin{array}{l}239 \\
(38.4)\end{array}$ & 83 (33.9) & $156(41.3)$ & \\
\hline & $26-50 \%$ & $\begin{array}{l}126 \\
(20.2)\end{array}$ & 48 (19.6) & 78 (20.6) & \\
\hline & $51-75 \%$ & $\begin{array}{l}132 \\
(21.2)\end{array}$ & 57 (23.3) & 75 (19.8) & \\
\hline & $76+\%$ & $\begin{array}{l}126 \\
(20.2)\end{array}$ & $57(23.3)$ & 69 (18.3) & \\
\hline \multirow[t]{6}{*}{ Ambient Noise } & Any data & $\begin{array}{l}581 \\
(93.3)\end{array}$ & $222(90.6)$ & $359(95.0)$ & \\
\hline & No data & $42(6.7)$ & $23(9.4)$ & $19(5.0)$ & \\
\hline & $0-25 \%$ & $\begin{array}{l}273 \\
(43.8)\end{array}$ & $100(40.8)$ & $173(45.8)$ & $\begin{array}{l}5.4 \\
(0.15)\end{array}$ \\
\hline & $26-50 \%$ & $\begin{array}{l}134 \\
(21.5)\end{array}$ & 47 (19.2) & $87(23.0)$ & \\
\hline & $51-75 \%$ & $\begin{array}{l}124 \\
(19.9)\end{array}$ & $58(23.7)$ & $66(17.5)$ & \\
\hline & $76+\%$ & $92(14.8)$ & $40(16.3)$ & $52(13.8)$ & \\
\hline \multirow[t]{6}{*}{ GPS Location } & Any data & $\begin{array}{l}603 \\
(96.8)\end{array}$ & 232 (94.7) & 371 (98.2) & \\
\hline & No data & $20(3.2)$ & $13(5.3)$ & $7(1.8)$ & \\
\hline & $0-25 \%$ & $\begin{array}{l}246 \\
(39.5)\end{array}$ & 85 (34.7) & $161(42.6)$ & $\begin{array}{l}5.3 \\
(0.15)\end{array}$ \\
\hline & $26-50 \%$ & $\begin{array}{l}133 \\
(21.3)\end{array}$ & $51(20.8)$ & $82(21.7)$ & \\
\hline & $51-75 \%$ & $\begin{array}{l}129 \\
(20.7)\end{array}$ & $58(23.7)$ & $71(18.8)$ & \\
\hline & $76+\%$ & $\begin{array}{l}115 \\
(18.5)\end{array}$ & $51(20.8)$ & $64(16.9)$ & \\
\hline Bluetooth & Any data & 596 & 231 (94.3) & 365 (96.6) & \\
\hline
\end{tabular}




\begin{tabular}{|c|c|c|c|c|c|}
\hline & \multirow{3}{*}{$\begin{array}{l}\text { No data } \\
0-25 \%\end{array}$} & \multirow{3}{*}{$\begin{array}{l}27(4.3) \\
237 \\
(38.0)\end{array}$} & \multirow{3}{*}{$\begin{array}{l}14(5.7) \\
82(33.5)\end{array}$} & \multirow{2}{*}{\multicolumn{2}{|c|}{$13(3.4)$}} \\
\hline & & & & & \\
\hline & & & & $155(41.0)$ & $\begin{array}{l}5.4 \\
(0.15)\end{array}$ \\
\hline & $26-50 \%$ & $\begin{array}{l}128 \\
(20.5)\end{array}$ & 48 (19.6) & $80(21.2)$ & \\
\hline & $51-75 \%$ & $\begin{array}{l}133 \\
(21.3)\end{array}$ & $59(24.1)$ & 74 (19.6) & \\
\hline & $76+\%$ & $\begin{array}{l}125 \\
(20.1)\end{array}$ & $56(22.9)$ & 69 (18.3) & \\
\hline \multirow[t]{6}{*}{ App Use } & Any data & $\begin{array}{l}579 \\
(92.9)\end{array}$ & 225 (91.8) & 354 (93.7) & \\
\hline & No data & $44(7.1)$ & $20(8.2)$ & $24(6.3)$ & \\
\hline & $0-25 \%$ & $\begin{array}{l}240 \\
(38.5)\end{array}$ & $82(33.5)$ & $158(41.8)$ & $\begin{array}{l}9.8 \\
(0.02)\end{array}$ \\
\hline & $26-50 \%$ & $\begin{array}{l}133 \\
(21.3)\end{array}$ & $46(18.8)$ & $87(23.0)$ & \\
\hline & $51-75 \%$ & $\begin{array}{l}122 \\
(19.6)\end{array}$ & $58(23.7)$ & $64(16.9)$ & \\
\hline & $76+\%$ & $\begin{array}{l}128 \\
(20.5)\end{array}$ & $59(24.1)$ & 69 (18.3) & \\
\hline \multirow[t]{6}{*}{$\begin{array}{l}\text { Phone } \\
\text { interactions }\end{array}$} & Any data & $\begin{array}{l}584 \\
(93.7)\end{array}$ & $225(9.2)$ & 359 (95.0 & \\
\hline & No data & $39(6.3)$ & $20(8.9)$ & $19(5.0)$ & \\
\hline & $0-25 \%$ & $\begin{array}{l}237 \\
(38.0)\end{array}$ & 83 (33.9) & $154(40.7)$ & $\begin{array}{l}8.8 \\
(0.03)\end{array}$ \\
\hline & $26-50 \%$ & $\begin{array}{l}136 \\
(21.8)\end{array}$ & 46 (18.8) & $90(23.8)$ & \\
\hline & $51-75 \%$ & $\begin{array}{l}119 \\
(19.1)\end{array}$ & $55(22.5)$ & 64 (16.9) & \\
\hline & $76+\%$ & $\begin{array}{l}131 \\
(21.0)\end{array}$ & $61(24.9)$ & 70 (18.5) & \\
\hline
\end{tabular}

${ }^{*}$ Calculated as the total number of days in which at least one data point has been provided. ${ }^{* \star}$ App names, foreground or background app use, time spent using apps. ${ }^{*} *$ How individuals interact with their phones, including phone screen on time, number of interactions with keyboard, screen touches, and extent to which the phone is asleep or awake. 
Figures

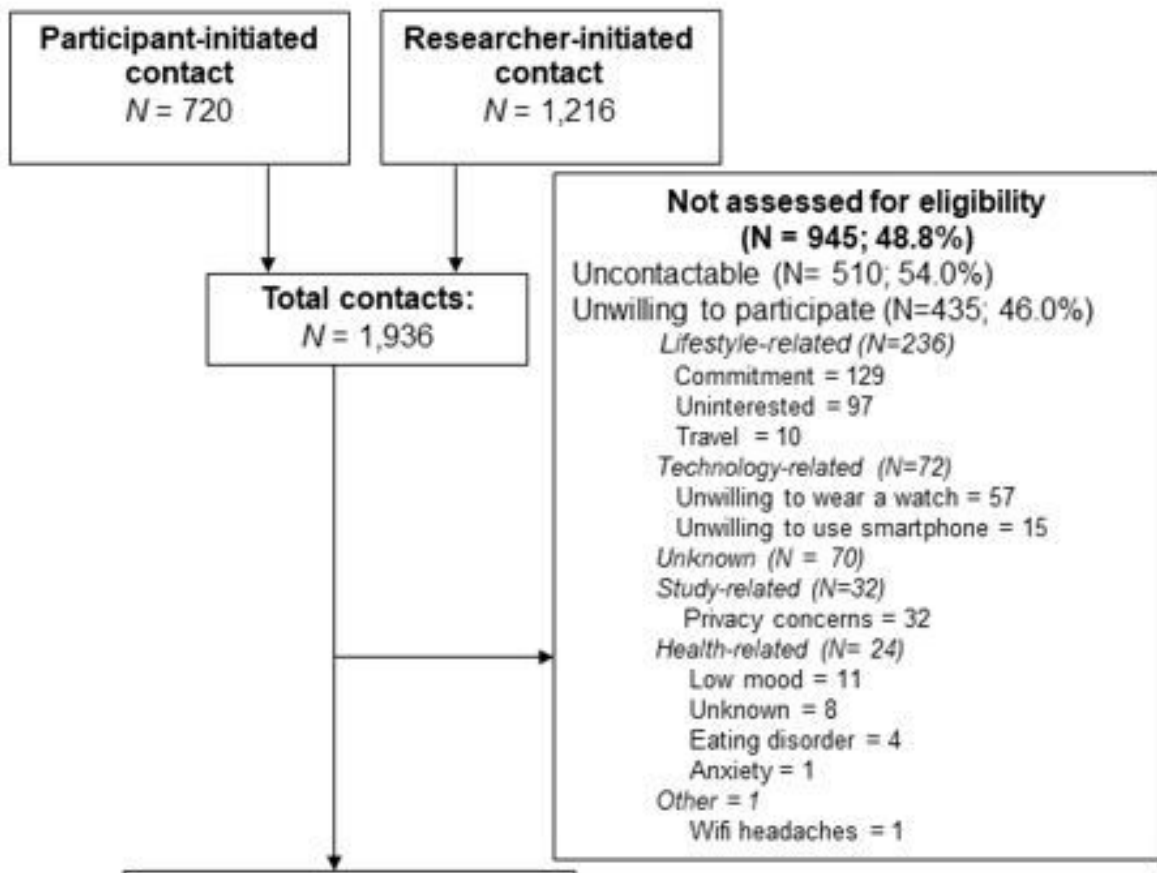

Total willing \& assessed for

eligibility

$(\mathrm{N}=991 ; 51.2 \%)$
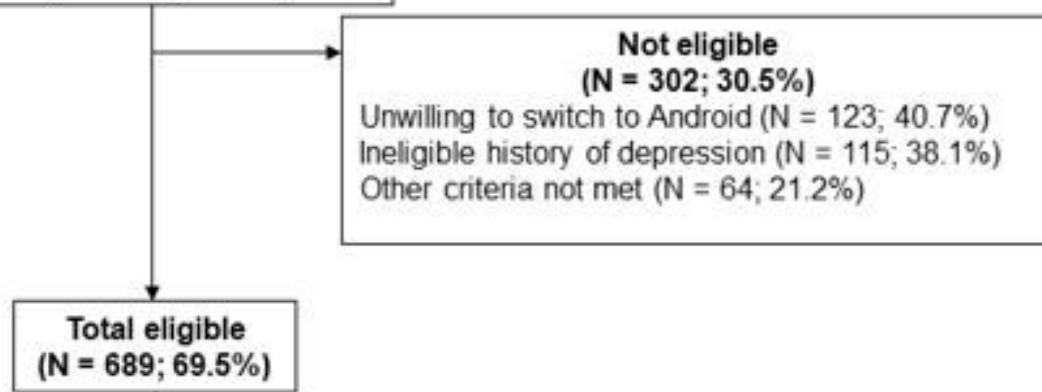

* Total enrolled $=62.8 \%$ of total willing and assessed for eligibility and $32.1 \%$ of total contacts.

\section{Figure 1}

Recruitment into RADAR-MDD 


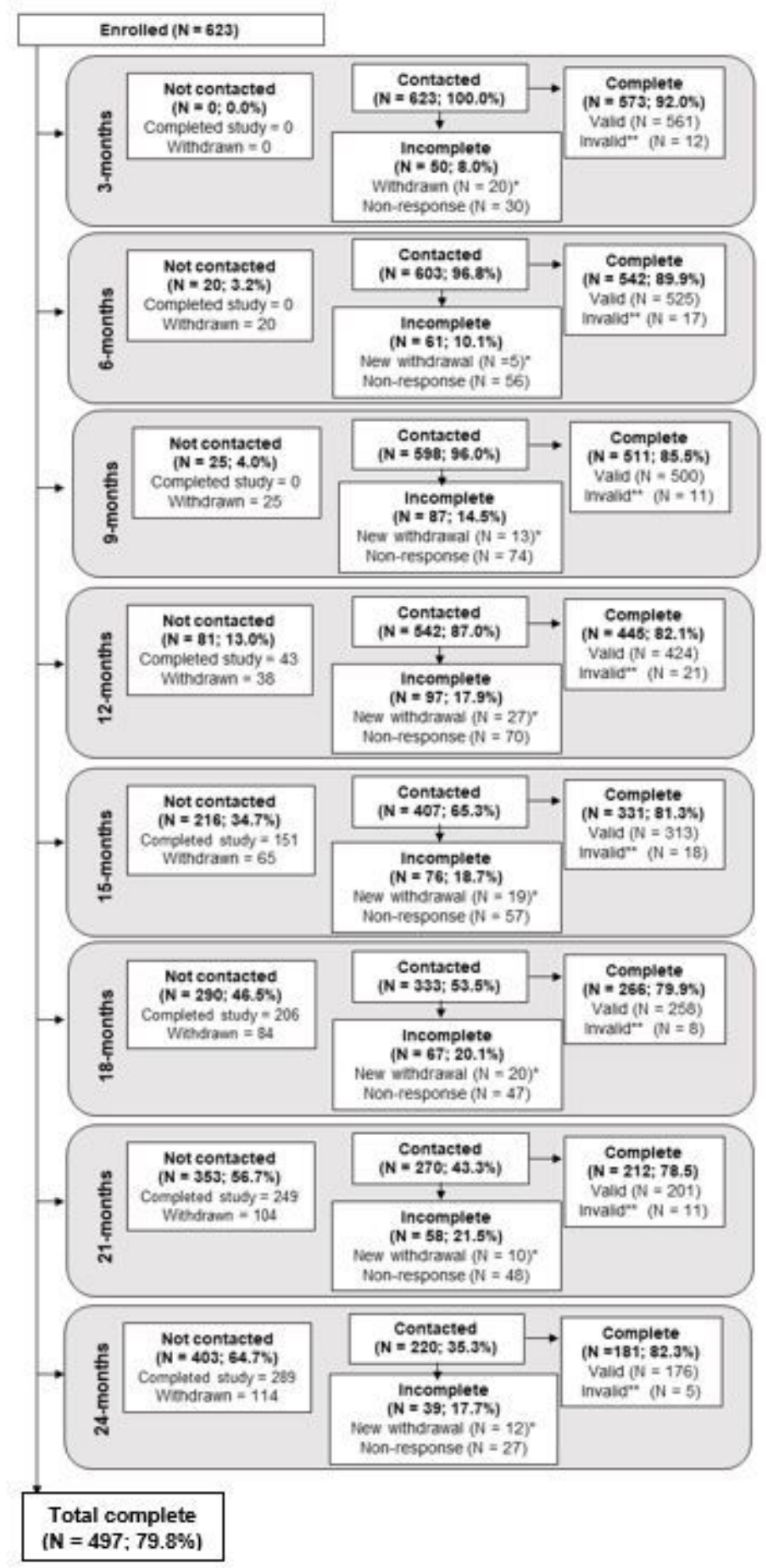

Figure 2

Retention of participants throughout follow-up. 
Figure 3a. PHQ8

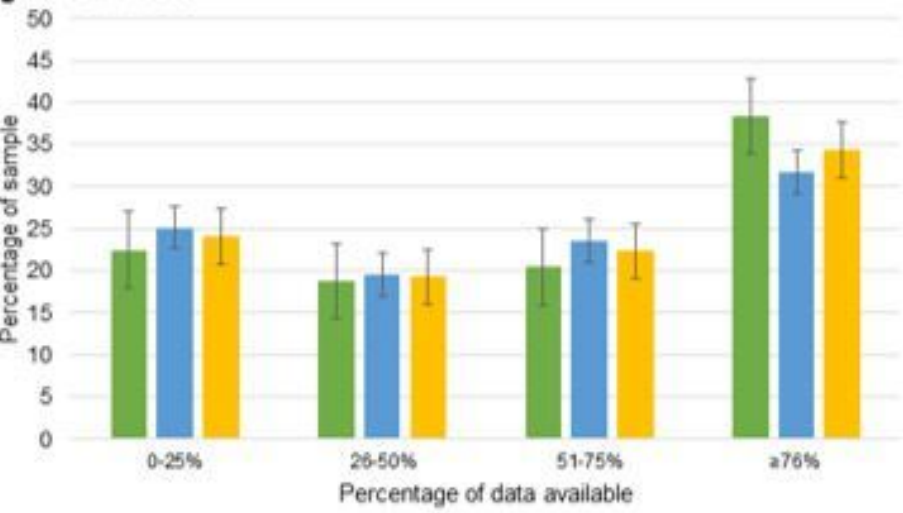

$=$ Not depressed $(\mathrm{N}=245) \quad=$ Depressed $(\mathrm{N}=378) \quad=$ Entire sample $(\mathrm{N}=623)$

Figure 3b. RSES

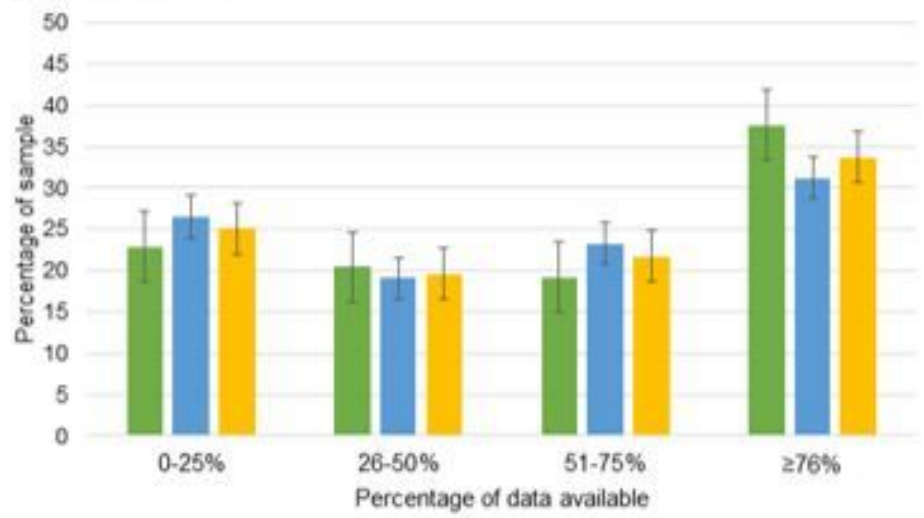

$=$ Not depressed $(\mathrm{N}=245) \quad=$ Depressed $(\mathrm{N}=378)=$ Entire sample $(\mathrm{N}=623)$

Figure 3c. Speech task

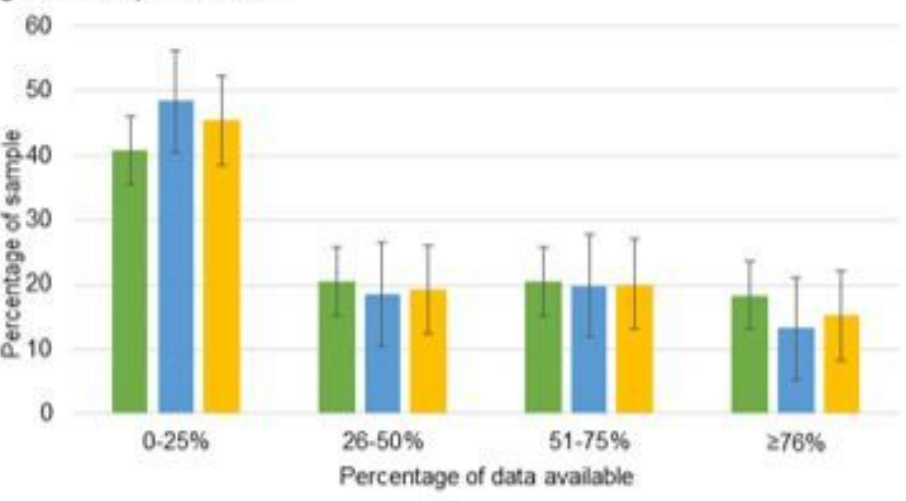

$=$ Not depressed $(\mathrm{N}=245) \quad=$ Depressed $(\mathrm{N}=378)=$ Entire sample $(\mathrm{N}=623)$

\section{Figure 3}

Illustration of data collected via RADAR-base aRMT app 
Figure 4a. PDQ5

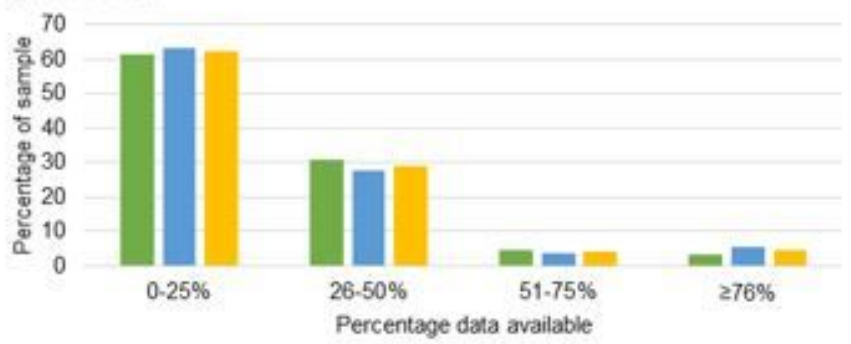

Figure $4 b$. Code breaker

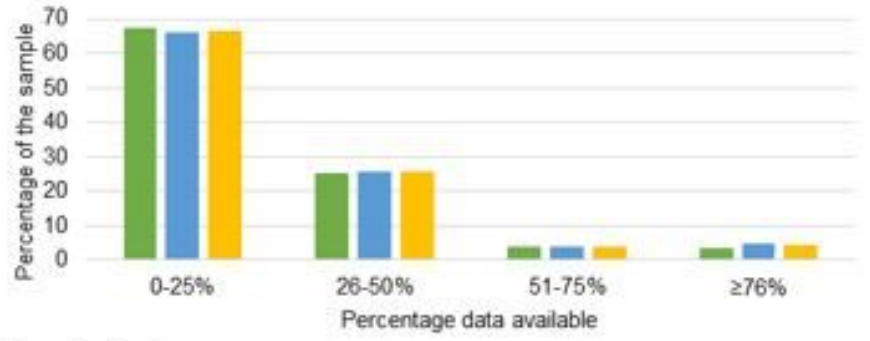

Figure $4 c$. Spotter

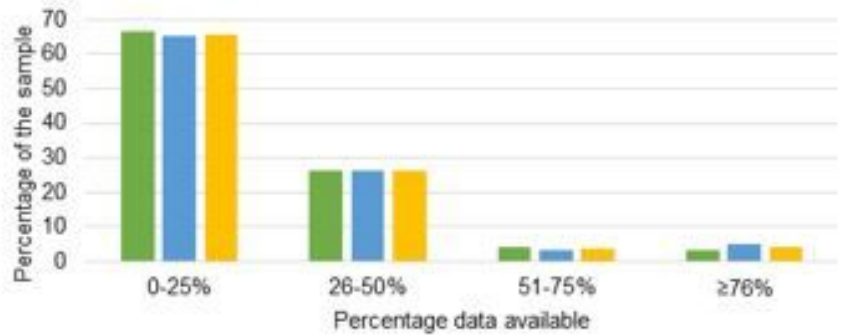

Figure 4d. Symbol Check
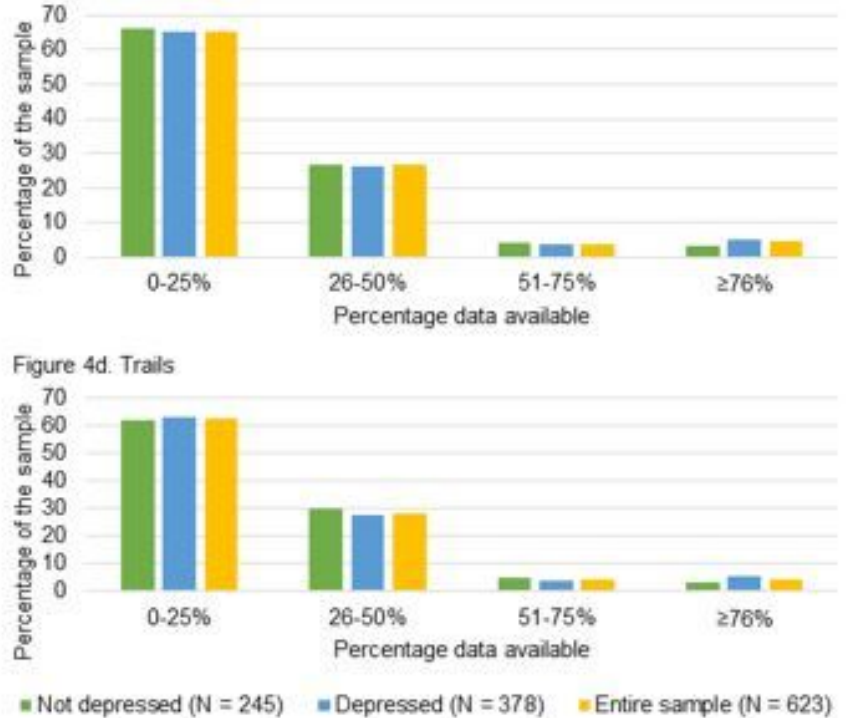

\section{Figure 4}

Illustration of data collected via THINC-it app 

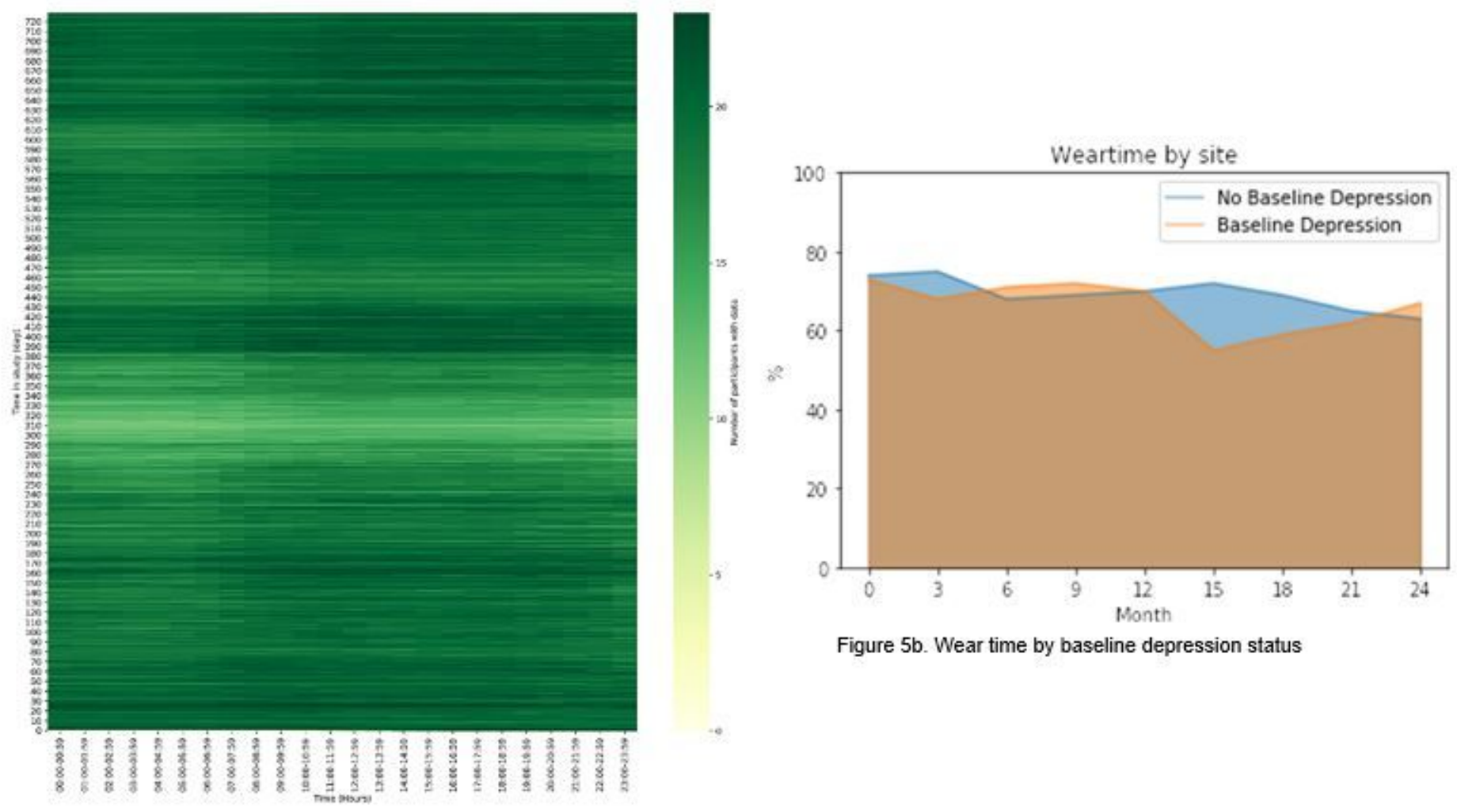

Figure $5 b$. Wear time by baseline depression status

\section{Figure 5}

Illustration of Fitbit data streams.

\section{Supplementary Files}

This is a list of supplementary files associated with this preprint. Click to download.

- Table1v1.0.docx

- AdditionalFiles.docx

- AdditionalFiles.docx 